\title{
THE SERIAL GORRELATION COEFFIGIENTS OF WAITING TIMES IN A STATIONARY SINGLE SERVER QUEUE
}

\author{
D. J. DALEY
}

(Received 3 January 1967)

\section{Summary}

In a stationary $G I / G / 1$ queueing system in which the waiting time variance is finite, it can be shown that the serial correlation coefficients $\left\{\rho_{n}\right\}$ of a (stationary) sequence of waiting times are non-negative and decrease monotonically to zero. By means of renewal theory we find a representation for $\sum_{0}^{\infty} \rho_{n}$ from which necessary and sufficient conditions for its finiteness can be found. In $M / G / 1$ rather more can be said: $\left\{\rho_{n}\right\}$ is a convex sequence, the asymptotic form of $\rho_{n}$ can be given in a nearly saturated queue, and a simple explicit expression for $\sum_{0}^{\infty} \rho_{n}$ exists. For the stationary $M / M / 1$ queue we find the $\rho_{n}$ 's explicitly, illustrate them numerically, and derive a representation which shows that $\left\{\rho_{n}\right\}$ is completely monotonic.

\section{Introduction}

We are concerned in this paper with a single server first-come firstserved queueing system with service times $\left\{S_{t}\right\}(t=0, \pm 1, \cdots)$ and interarrival times $\left\{T_{t}\right\}$ mutually independent, and each identically and independently distributed with distribution functions (d.f.s.) $B(x)=P\left[S_{t} \leqq x\right]$ and $A(x)=P\left[T_{t} \leqq x\right]$ respectively. If $E\left[U_{t}\right]=E\left[S_{t}-T_{t}\right]<0$, then it is well known that a stationary distribution for the waiting times $\left\{W_{t}\right\}$ exists, with $W(x)=P\left[W_{t} \leqq x\right]$ (all $t$ ) satisfying the Wiener-Hopf equation

$$
W(x)= \begin{cases}\int_{0-}^{\infty} U(x-y) d W(y) & (x \geqq 0), \\ 0 & (x<0),\end{cases}
$$

where $U(x)=P\left[U_{t} \leqq x\right]=\int_{0}^{\infty} B(x+y) d A(y)(-\infty<x<\infty)$. Here and throughout the paper, $\left\{W_{t}\right\}$ denotes a sequence of waiting times in such a stationary $G I / G / 1$ queue, namely, one for which $E\left[U_{t}\right]<0$. Kiefer and Wolfowitz [11] showed that a sufficient condition for $E\left[W_{t}^{r}\right]=\int_{0}^{\infty} x^{r} d W(x)$ to be finite is that $\mu_{r+1}=\int_{0}^{\infty} x^{r+1} d B(x)$ should be finite, and that this 
condition is also necessary if $\int_{0}^{\infty} x d A(x)$ is finite. Since we shall be discussing the behaviour of $\left\{\rho_{n}\right\}$ where

$$
\begin{array}{ll}
\rho_{n}=\gamma_{n} / \sigma^{2} & (n=0,1, \cdots), \\
\gamma_{n}=E\left[\left(W_{t}-\mu\right)\left(W_{t+n}-\mu\right)\right] & (\text { all } n \text { and } t), \\
\mu=E\left[W_{t}\right] \text { and } \sigma^{2}=E\left[W_{t}^{2}\right]-\mu^{2} & (\text { all } t),
\end{array}
$$

(if all these quantities are finite), we shall assume throughout that

$$
\mu_{3}=E\left[S_{t}^{3}\right]=\int_{0}^{\infty} x^{3} d B(x)<\infty .
$$

We also assume that $E\left[T_{t}\right]=\int_{0}^{\infty} x d A(x)<\infty$. (The independence of $\gamma_{n}, \mu$, and $\sigma^{2}$ of $t$ follows from the stationarity of $\left\{W_{t}\right\}$.) For simplicity we omit the suffix $t$ in expectations where no confusion may arise; for example, $\sigma^{2}=E\left[W^{2}\right]-(E[W])^{2}$ and $\mu_{3}=E\left[S^{3}\right]$.

The motivation for this work is twofold. In the first place, it is a standard result in the analysis of a stationary time series $\left\{X_{t}\right\}$ that, given an observed sequence $\left\{x_{1}, \cdots, x_{N}\right\}$ and setting $\bar{x}=\left(x_{1}+\cdots+x_{N}\right) / N$, a large sample approximation to $N$ var $(\bar{x})$ is

$$
\operatorname{var}\left(X_{0}\right)+2 \sum_{1}^{\infty} \operatorname{cov}\left(X_{0}, X_{n}\right)=\operatorname{var}\left(X_{0}\right)\left(1+2 \sum_{1}^{\infty} \rho_{n}\right)
$$

where $\rho_{n}$ is the $n^{\text {th }}$ serial correlation coefficient. Such waiting time sequences $\left\{W_{t}\right\}$ as we are discussing fall into this framework, and in Theorem 2 below an expression for $\sum_{0}^{\infty} \rho_{n}$ is developed from which both its approximate value and conditions for its finiteness can be found. An alternative method (in principle anyway) of finding $\sum_{0}^{\infty} \rho_{n}$ via the solution of an integral equation may be deduced as a corollary. Not surprisingly, $\sum_{0}^{\infty} \rho_{n}$ can be found exactly in explicit form in a stationary $M / G / 1$ queueing system (Theorem 4). This aspect of the paper is related to Breny's studies $[2,3]$ on large sample estimates for the mean queue size in $M / M / 1$, with which Jackson's Monte Carlo work $[10]$ is linked. Morse ([15]; see also Saaty [18] p. 130) derived the autocorrelation function of the continuous time queue size process in $M / M / 1$ (with comments on $M / M / 2$ and $M / M / 3$ ), and Fishman and Kiviat [9] used Morse's work in a simulation study of spectral analyses of time series derived from fairly simple queueing systems. Work on similar problems in pure loss systems (i.e., with no waiting facilities) seems more extensive (e.g. $[1],[14]$ pp. 190-200, [17] chapter 6).

In other respects this paper is a development of Craven's [6] study concerning the serial dependence of Markov chains. Craven cites as an example the second order properties of waiting times in the queue $M / M / 1$, conjecturing that for each $n$, the correlation coefficients $\rho_{n}$ satisfy

$$
\rho_{n}=1-n(1-\tau)^{2}+O\left((1-\tau)^{3}\right)
$$


for $\tau=E[S] / E[T] \uparrow 1$. A generalized form of (1) is proved in Theorem 5 for the system $M / G / 1$, and by comparing this with other heavy traffic results of Kingman [12], this leads to a conjecture of the analogue of (1) in the system $G I / G / 1$.

The results in Sections 2 and 3 below lead to considering in [7] Markov chains more general than $\left\{W_{t}\right\}$ : this paper is in one sense an extended example of that work. It illustrates moreover some of the rationale behind [6]: there is a vast general theory for the (numerical) analysis of second order stationary time series on the one hand, and an extensive discussion of the simplest non-trivial stochastic processes (namely, Markov chains) on the other, yet it seems the two have rarely been fused.

\section{The monotonic character of $\left\{\rho_{n}\right\}$ in $G I / G / 1$}

THEOREM 1. If $\left\{W_{t}\right\}$ is a sequence of waiting times for the stationary queue $G I / G / 1$ with $\mu_{3}=E\left[S^{3}\right]=\int_{0}^{\infty} x^{3} d B(x)<\infty$, then the serial correlation coefficients $\left\{\rho_{n}\right\}$ decrease monotonically to zero.

Proof. A more general theorem of which the present result is a special case is given elsewhere [7]. However, the notation and an auxiliary result are wanted later, so the formal algebraic steps in the proof are given here. Equations (2), (3), and (4) below are essentially in [6], but the generality claimed in [6] for $\lim _{n \rightarrow \infty} C_{n}(x)$ is not true. The notation in (2) below is an important simplification (cf. [21]).

The chief point is to define the sequence of functions

$$
C_{n}(x)=\int_{W_{n} \leqq x} W_{0} P\left(d W_{0}, d W_{n}\right)=\int_{0 \leqq W_{n} \leqq x} W_{0} P\left(d W_{0}, d W_{n}\right)
$$

where $\boldsymbol{P}(\cdot)$ denotes the probability measure associated with the stationary sequence $\left\{W_{t}\right\}$. These functions can be shown to satisfy

$$
\rho_{n} \sigma^{2}+\mu^{2}=\int_{0}^{\infty} x d C_{n}(x)=\int_{0}^{\infty}\left(\mu-C_{n}(x)\right) d x
$$

and

$$
C_{n+1}(x)=\int_{0-}^{\infty} U(x-y) d C_{n}(y)=\int_{-\infty}^{x} C_{n}(x-y) d U(y),
$$

from which we deduce that

$$
\left(\rho_{n}-\rho_{n+1}\right) \sigma^{2}=\int_{0}^{\infty} H_{n}(x) d x
$$

and

$$
H_{n+1}(x)=\int_{-\infty}^{x} H_{n}(x-y) d U(y),
$$

where $H_{n}(x)=C_{n+1}(x)-C_{n}(x)$. Thus, if $H_{0}(x)$ can be shown to be non- 
negative for all $x \geqq 0$, it will follow that (i) every $H_{n}(\cdot)$ is a non-negative function, and therefore $\rho_{n} \geqq \rho_{n+1}$; and (ii) for every fixed $x \geqq 0$, and in particular for $x=0,\left\{C_{n}(x)\right\}$ is a non-decreasing sequence. To prove that $H_{0}(\cdot) \geqq 0$, we first use integration by parts in the equation

$$
\begin{aligned}
C_{1}(y)-C_{0}(y) & =\int_{0}^{\infty} x d_{x} \boldsymbol{P}\left[W_{0} \leqq x, W_{1} \leqq y\right]-\int_{0}^{\infty} x d_{x} \boldsymbol{P}\left[W_{0} \leqq x, W_{0} \leqq y\right] \\
& =\int_{0}^{\infty}\left(\boldsymbol{P}\left[W_{0} \leqq \min (x, y)\right]-\boldsymbol{P}\left[W_{0} \leqq x, W_{1} \leqq y\right]\right) d x,
\end{aligned}
$$

and then observe that by stationarity of $\left\{W_{t}\right\}$,

$$
\begin{aligned}
\boldsymbol{P}\left[W_{0} \leqq x, W_{1} \leqq y\right] & \leqq \min \left(\boldsymbol{P}\left[W_{0} \leqq x\right], \boldsymbol{P}\left[W_{1} \leqq y\right]\right) \\
& =\boldsymbol{P}\left[W_{\mathbf{0}} \leqq \min (x, y)\right],
\end{aligned}
$$

so the last integrand is non-negative. Using now Lindley's uniqueness theorem for probabilistic solutions of the Wiener-Hopf equation, we can deduce that $\lim _{n \rightarrow \infty} C_{n}(x)=\mu W(x)$. By monotone convergence and (3), $\lim _{n \rightarrow \infty} \rho_{n}=0$.

The properties of $\left\{C_{n}(\cdot)\right\}$ are usefully stated as

LEMMA 1. For each fixed $x \geqq 0$,

$$
0 \leqq C_{n}(x) \leqq C_{n+1}(x) \rightarrow \mu W(x) \quad(n \rightarrow \infty) .
$$

\section{Conditions for the finiteness of $\sum_{0}^{\infty} \rho_{n}$ and its evaluation}

Referring to (3) and recalling that $\mu=E[W]=\int_{0}^{\infty}(1-W(x)) d x$, consider for $y \geqq 0$ and $n=0,1, \cdots$, the functions $\left\{\gamma_{n}(\cdot)\right\}$ and $\left\{p_{n}(\cdot)\right\}$ defined by

$$
\begin{aligned}
\gamma_{n}(y) & =\sigma^{2} \rho_{n}(y)=\int_{0}^{y}\left(\mu W(x)-C_{n}(x)\right) d x & (y \geqq 0) \\
& =\int_{0}^{y}\left(\mu-C_{n}(x)\right) d x-\mu \int_{0}^{y}(1-W(x)) d x, &
\end{aligned}
$$

so that $\gamma_{n}=\lim _{y \rightarrow \infty} \gamma_{n}(y)$. Then since $W(x)=\int_{-\infty}^{x} W(x-y) d U(y)$ for $x \geqq 0$, and using (4),

$$
\begin{aligned}
\gamma_{n+1}(y) & =\int_{0}^{y} d x\left(\int_{-\infty}^{x} \mu W(x-u) d U(u)-\int_{-\infty}^{x} C_{n}(x-u) d U(u)\right) \\
& =\int_{-\infty}^{y} d U(u) \int_{\max (0, u)}^{y}\left(\mu W(x-u)-C_{n}(x-u)\right) d x \\
& =\int_{-\infty}^{y}\left(\gamma_{n}(y-u)-\gamma_{n}(\max (-u, 0))\right) d U(u) .
\end{aligned}
$$

Equivalently,

$$
\rho_{n+1}(y)=\int_{-\infty}^{y}\left(\rho_{n}(y-u)-\rho_{n}(\max (-u, 0))\right) d U(u) .
$$


By Lemma 1, $C_{n}(x)$ converges monotonically from below to $\mu W(x)$ for all $x \geqq 0$, so (7) shows that $\rho_{n}(y)$ is for each $n$ a continuous non-decreasing function of $y$, with $\rho_{n}(0)=0$ and $\lim _{y \rightarrow \infty} \rho_{n}(y)=\rho_{n} \leqq 1$. Thus, each $\rho_{n}(\cdot)$ is the d.f. of a (possibly dishonest) positive random variable. Specifically, given a sequence $\left\{U_{n}\right\}(n=1,2, \cdots)$ of independent random variables with common d.f. $U(\cdot)$, and given a positive random variable $Z_{0}$ with (honest) d.f. $\rho_{0}(y)=P\left[Z_{0} \leqq y\right]$, we deduce from (9) that the random variables of the sequence $\left\{Z_{n}\right\}$ defined for $n=1,2, \cdots$ by

$$
Z_{n}= \begin{cases}Z_{n-1}+U_{n} & \text { if } 0<Z_{n-1}+U_{n}<\infty, \\ \infty & \text { if otherwise, }\end{cases}
$$

have as their d.f.s. $\rho_{n}(y)=P\left[Z_{n} \leqq y\right]$. (In (10), $Z_{n-1}=\infty$ implies $Z_{n}=\infty$ with probability one because $U_{n}$ is finite with probability one.)

Thus, given $Z_{0}=z$ say $(z>0)$, we have $0<Z_{n}<\infty$ if and only if every partial sum $z-S_{1}, \cdots, z-S_{n}$ is positive, where $S_{m}=-\sum_{r=1}^{m} U_{r}$, i.e., setting $M_{n}=\max \left(0, S_{1}, \cdots, S_{n}\right)$,

$$
P\left[0<Z_{n}<\infty \mid Z_{0}=z\right]=P\left[M_{n}<z\right] .
$$

From the integral equation of renewal theory, we have for $0<z<\infty$ that

$$
0 \leqq \sum_{n=1}^{\infty} P\left[M_{n}<z\right]=H(z-0) \leqq \sum_{n=1}^{\infty} P\left[M_{n} \leqq z\right]=H(z)<\infty,
$$

where $H(\cdot)$ is the renewal function satisfying the renewal equation ${ }^{1}$

$$
H(z)= \begin{cases}V(z)+\int_{-\infty}^{z} H(z-y) d V(y) & (z \geqq 0), \\ 0 & (z<0),\end{cases}
$$

in which $V(y)=1-U(-y-0)$ is the d.f. of the random variables of the sequence $\left\{-U_{n}\right\}$; the finiteness of $H(z)$ comes from $E[-U]>0$, $E[|U|]<\infty$. Now

$$
\rho_{n}=P\left[0<Z_{n}<\infty\right]=\int_{0}^{\infty} P\left[0<Z_{n}<\infty \mid Z_{0}=z\right] d \rho_{0}(z),
$$

so

$$
\sum_{n=1}^{\infty} \rho_{n}=\sum_{n=1}^{\infty} \int_{0}^{\infty} P\left[0<Z_{n}<\infty \mid Z_{0}=z\right] d \rho_{0}(z)=\int_{0}^{\infty} H(z-0) d \rho_{0}(z),
$$

where equality holds in the sense that both sides converge or diverge together. Since by $(7) \rho_{0}(\cdot)$ is absolutely continuous (indeed, its derivative is continuous), and the inequality $H(z-0)<H(z)$ can hold for at most a countable set of values of $z$,

${ }^{1} H(\cdot)$ is in fact the unique finite solution of the equation (see [8] pp. 382-385). 


$$
\sum_{n=1}^{\infty} \rho_{n}=\int_{0}^{\infty} H(z) d \rho_{0}(z)
$$

Now it is easy to modify the renewal theorem (e.g. [8], chapters XI and XII in particular) to deduce that for all $z \geqq 0$,

$$
\frac{1}{2} c z+C_{1} \leqq H(z) \leqq 2 c z+C_{2}
$$

where $C_{1}$ and $C_{2}$ are (finite) constants and $c^{-1}=E[-U]$ which implies that $c$ is finite and positive because we assumed at the outset that

$$
-\infty<E[S]-E[T]=E[U]<0 .
$$

Therefore the left hand side of (11) converges or diverges with $\int_{0}^{\infty} z d \rho_{0}(z)$. From (2) and (7),

$$
\begin{aligned}
\sigma^{2} \int_{0}^{\infty} y d \rho_{0}(y) & =\int_{0}^{\infty} y d \gamma_{0}(y)=\int_{0}^{\infty} y\left(\mu W(y)-C_{0}(y)\right) d y \\
& =\int_{0}^{\infty} y d y \int_{0-}^{y}(\mu-x) d W(x) \\
& =\int_{0}^{\infty} y d y \int_{y}^{\infty}(x-\mu) d W(x) \text { because } \int_{0-}^{\infty}(\mu-x) d W(x)=0 \\
& =\int_{0-}^{\infty} \frac{1}{2}\left(x^{3}-\mu x^{2}\right) d W(x) \text { on inverting the order of integration } \\
& =\frac{1}{2}\left(E\left[W^{3}\right]-E[W] E\left[W^{2}\right]\right),
\end{aligned}
$$

which is finite or infinite as $E\left[W^{3}\right]$ is finite or infinite: the inversion of the order of integration is justified by observing that the coefficient of $-\mu / 2$ equals $E\left[W^{2}\right]$ and is finite by assumption, while the remaining term has a non-negative integrand and is with respect to a non-negative measure, so Fubini's theorem applies. By the result of Kiefer and Wolfowitz [11] quoted in the introduction, if $E[T]<\infty$, the necessary and sufficient condition that $E\left[W^{3}\right]$ be finite is that $\mu_{4}=E\left[S^{4}\right]$ be finite. We have thus completed the proof of

THEOREM 2. If $\left\{W_{t}\right\}$ is a sequence of waiting times for the stationary queue $G I / G / 1$, with $\mu_{3}=E\left[S^{3}\right]<\infty, U(x)=P[U \leqq x],-\infty<E[U]<0$, and $E[|U|]<\infty$, then the necessary and sufficient condition that the sum $\sum_{0}^{\infty} \gamma_{n}$ of the serial covariances $\gamma_{n}=\operatorname{cov}\left(W_{t}, W_{t+n}\right)$ should be finite is that $\mu_{4}=E\left[S^{4}\right]<\infty$. Further

$$
\sum_{n=1}^{\infty} \gamma_{n}=\int_{0}^{\infty} H(x) d \gamma_{0}(x)=\int_{0}^{\infty} H(x) d x \int_{0-}^{x}(\mu-y) d W(y)
$$

(equality holding in the sense that both sides may diverge) where

$$
\gamma_{0}(y)=\int_{0}^{v}\left(\mu W(x)-C_{0}(x)\right) d x=\int_{0-}^{v}(y-x)(\mu-x) d W(x)
$$

and $H(x)$ is the unique finite non-negative solution of 


$$
\begin{aligned}
& H(x)=V(x)+\int_{-\infty}^{x} H(x-y) d V(y) \quad(x \geqq 0), \\
& V(x)=1-U(-x-0) .
\end{aligned}
$$

An alternative possible method of evaluating $\sum_{0}^{\infty} \rho_{n}$ is to deduce from (9) an integral equation for $R(y)=\sum_{0}^{\infty} \rho_{n}(y)$ and finding $\lim _{y \rightarrow \infty} R(y)$.

Intuitively it is obvious from (12) and (13) that an approximation to $\sum_{1}^{\infty} \gamma_{n}$ (when finite) is

$$
\sum_{n=1}^{\infty} \gamma_{n} \approx \frac{E\left[W^{3}\right]-E[W] E\left[W^{2}\right]}{2 E[-U]}
$$

Kingman $[12,13]$ has shown that for $\kappa E[-U]$ small, where $\kappa=2 E[-U] / \operatorname{var}(U)$ (assuming of course that $\operatorname{var}(U)<\infty$ ), the stationary distribution $W(\cdot)$ is approximately negative exponential with mean $\kappa^{-1}$. If we assume that under the same conditions this implies that the second and third moments of $W(\cdot)$ are approximately equal to $2 \kappa^{-2}$ and $6 \kappa^{-3}$ respectively, then (14) would suggest that $\sum_{1}^{\infty} \gamma_{n} \approx 2 \kappa^{-3} / E[-U]$, and so

$$
\sum_{n=-\infty}^{\infty} \rho_{n} \approx 1+\frac{2 \operatorname{var}(U)}{(E[-U])^{2}} .
$$

We do not propose investigating these assumptions here, though comparison of (15) with the exact expression at (28) below for $M / G / 1$ suggests that it is in that case an asymptotic result for $\kappa \downarrow 0$.

\section{Serial correlation coefficients of waiting times in $M / G / 1$}

The techniques used in this section to find explicit expressions for $\left\{\rho_{n}\right\}$ in the stationary queue $M / G / 1$ are essentially standard in queueing theory. We assume a Poisson arrival process with rate $\lambda$, and denote the Laplace-Stieltjes transforms of $B(\cdot)$ and $W(\cdot)$ by $\beta(\theta)=\int_{0}^{\infty} e^{-\theta x} d B(x)$ and $\varphi(\theta)=\int_{0-}^{\infty} e^{-\theta x} d W(x)(R l(\theta) \geqq 0)$. Then (e.g. [16] p. 42) for $R l(\theta)>0$, $\varphi(\theta)$ and $\beta(\theta)$ are related by the Pollaczek-Khintchine equation

$$
\varphi(\theta)=\frac{(1-\tau) \theta}{\theta-\lambda(1-\beta(\theta))}
$$

where, with $\mu_{r}=\int_{0}^{\infty} x^{r} d B(x)(r=1,2,3,4), \tau=\lambda \mu_{1}$ is the traffic intensity. The mean $\mu$ and the variance $\sigma^{2}$ of the process $\left\{W_{t}\right\}$ are easily deduced from (16) to be

$$
\mu=\frac{\lambda \mu_{2}}{2(1-\tau)}, \quad \sigma^{2}=\frac{\lambda^{2} \mu_{2}^{2}}{4(1-\tau)^{2}}+\frac{\lambda \mu_{3}}{3(1-\tau)} .
$$

With this notation we shall prove 
THEOREM 3. The serial correlation coefficients $\left\{\rho_{n}\right\}$ of the waiting time sequence $\left\{W_{t}\right\}$ in the stationary queue $M / G / 1$ are given by

$$
\rho_{n}=1-n \mu(1-\tau) / \lambda \sigma^{2}+\left(\sum_{r=1}^{n} C_{r}\right) / \lambda \sigma^{2} \quad(n=1,2, \cdots)
$$

where with $D \equiv d / d \theta$,

$$
\begin{aligned}
C_{r} & =\int_{W_{r}=0} W_{0} P\left(d W_{0}, d W_{r}\right) \\
& =\left\{\begin{array}{rr}
-\beta(\lambda) \varphi^{\prime}(\lambda) & (r=1), \\
\frac{1}{r !}\left[D^{r-2}\left(r[-\lambda \beta(\theta)]^{r} D\left(\frac{\varphi^{\prime}(\theta)}{\theta}\right)+\left(\frac{\varphi^{\prime}(\theta)}{\theta}\right) D\left([-\lambda \beta(\theta)]^{r}\right)\right)\right. & ]_{\theta=\lambda} \\
& (r=2,3, \cdots) .
\end{array}\right.
\end{aligned}
$$

For $|z|<1$,

$$
\sum_{r=1}^{\infty} C_{r} z^{r}=\frac{(u(z)-\lambda) \varphi^{\prime}(u(z))}{u(z)}
$$

where $\theta=u(z)=g^{-1}(z)$ is the unique inverse from $0<z<1$ to $\lambda>\theta>0$ of

$$
z=\frac{\lambda-\theta}{\lambda \beta(\theta)} \equiv g(\theta)
$$

REMARK. It follows from the theorem that

$$
\lambda \sigma^{2}\left(\rho_{n}-\rho_{n+1}\right)=\mu(1-\tau)-C_{n+1}
$$

and so

$$
\lambda \sigma^{2}\left(\rho_{n}-2 \rho_{n+1}+\rho_{n+2}\right)=\lambda \sigma^{2} \Delta^{2} \rho_{n}=C_{n+2}-C_{n+1} .
$$

Lemma 1 states that $\left\{C_{n}\right\}=\left\{C_{n}(0)\right\}$ is a monotonic non-decreasing sequence, so it follows from (21) that in $M / G / 1,\left\{\rho_{n}\right\}$ is a convex sequence. $\left\{\rho_{n}\right\}$ being convex implies (theorem 5.1 .8 of [20]) that $\rho_{0}+2 \sum_{n=1}^{\infty} \rho_{n} \cos n x$ is convergent for $0<x \leqq \pi$ to a non-negative function $f(x)$ continuous on $(0, \pi]$ and integrable on $[0, \pi]$. Thus the spectral measure associated with the stationary process $\left\{W_{t}\right\}$ is absolutely continuous with the density function continuous except possibly at the origin. (In fact, the same is true knowing only that $\left\{\boldsymbol{p}_{n}\right\}$ is monotonic decreasing to zero, but the proof is more delicate and not directly relevant here.)

We shall show in Section 6 below that in $M / M / 1,\left\{\rho_{n}\right\}$ is a completely monotonic sequence, that is, $(-)^{r} \Delta^{r} \rho_{n} \geqq 0$ for all non-negative integers $r$ and $n$, where $\Delta^{r+1} \rho_{n}=\Delta^{r} \rho_{n+1}-\Delta^{r} \rho_{n}$.

Proof. The functions $\psi_{n}(\theta)=E\left[W_{0} e^{-\theta W_{n}}\right]$ exist for all $R l(\theta) \geqq 0$, and 


$$
\begin{aligned}
\psi_{n+1}(\theta) & =E\left[W_{0} e^{-\theta W_{n+1}}\right]=E\left[W_{0} \exp \left(-\theta\left(W_{n}+S_{n}-T_{n}\right)^{+}\right)\right] \\
& =E\left[E\left[W_{0} \exp \left(-\theta\left(W_{n}+S_{n}-T_{n}\right)^{+}\right) \mid W_{0}, W_{n}, S_{n}\right]\right] \\
& =\frac{E\left[\theta W_{0} e^{-\lambda\left(W_{n}+S_{n}\right)}-\lambda W_{0} e^{-\theta\left(W_{n}+S_{n}\right)}\right]}{\theta-\lambda}
\end{aligned}
$$

where we have used the fact that $T_{n}$ is negative exponential,

$$
P\left[T_{n} \geqq x\right]=e^{-\lambda x} \quad(x \geqq 0) .
$$

Thus

$$
\psi_{n+1}(\theta)=\frac{\theta \psi_{n}(\lambda) \beta(\lambda)-\lambda \psi_{n}(\theta) \beta(\theta)}{\theta-\lambda} \quad(\theta \neq \lambda)
$$

Introduce the generating function $\Psi(\theta, z)=\sum_{n=0}^{\infty} \psi_{n}(\theta) z^{n}(|z|<1)$; then for all $R l(\theta) \geqq 0, \theta \neq \lambda$, we deduce from (22) that

$$
\frac{\Psi(\theta, z)-\psi_{0}(\theta)}{z}=\frac{\theta \beta(\lambda) \Psi(\lambda, z)-\lambda \beta(\theta) \Psi(\theta, z)}{\theta-\lambda} .
$$

Now

$$
\lim _{\theta \rightarrow \infty} \psi_{n}(\theta)=\lim _{\theta \rightarrow \infty} \int_{0-}^{\infty} e^{-\theta x} d C_{n}(x)=C_{n}(0)=C_{n} \text { (say) }
$$

so putting $C(0, z)=\sum_{n=1}^{\infty} C_{n} z^{n}(|z|<1)$, it follows from (23) that $C(0, z)=z \beta(\lambda) \Psi(\lambda, z)$. Also, $\psi_{0}(\theta)=E\left[W_{0} e^{-\theta W_{0}}\right]=-\varphi^{\prime}(\theta)$, with $C_{0}=0$. We therefore obtain from (23) that for all $R l(\theta) \geqq 0$ (including $\theta=\lambda$ ),

$$
(\theta-\lambda+\lambda z \beta(\theta)) \Psi(\theta, z)=\theta C(0, z)-(\theta-\lambda) \varphi^{\prime}(\theta)
$$

(There is an alternative procedure available to deduce (24). Formally, it consists in defining the generating function

$$
C(x, z)=\sum_{n=0}^{\infty} C_{n}(x) z^{n}(|z|<1)
$$

and forming from (4) the equation for $C(x, z)$

$$
C(x, z)-C_{0}(x)=z \int_{0-}^{\infty} U(x-y) d_{y} C(y, z),
$$

which, assuming the differentiations below are justified, yields

$$
\left(\lambda-\frac{\partial}{\partial x}\right)\left(C(x, z)-C_{0}(x)\right)=\lambda z \int_{0-}^{x} B(x-y) d_{y} C(y, z),
$$

and hence (24) by taking Laplace transforms.)

For fixed $z$ in $(0,1)$, the function $f(\theta)=\theta+\lambda z \beta(\theta)-\lambda$ is a strictly monotonic increasing continuous function of $\theta$ on $\theta \geqq 0$, with

$$
f(0)=-\lambda(1-z)<0 \text { and } f(\lambda)=\lambda z \beta(\lambda)>0
$$


so for each $z$ in $(0,1)$, there is exactly one zero of $f(\theta)=0$ such that $0<\theta<\lambda$. Denote this zero by $\theta=u(z)$. Setting $z=(\lambda-\theta) / \lambda \beta(\theta)=g(\theta)$ say, this function $u(z)$ is the inverse function $g^{-1}$, mapping $0<z<1$ one-one onto $\lambda>\theta>0$, with $u(z) \uparrow \lambda$ for $z \downarrow 0$ and $u(z) \downarrow 0$ for $z \uparrow 1$. Also, $g(\theta)$ is analytic for $R l(\theta)>0$ and for $0<\theta<\lambda$ it has a non-vanishing derivative, so $u(z)$ is an analytic function of $z$ for $z$ in $(0,1)$ and since $\mu_{3}=-\lim _{\theta \downarrow 0} \beta^{\prime \prime \prime}(\theta)$ is assumed finite, it follows that $\lim _{z \uparrow 1} u^{\prime \prime \prime}(z)$ exists and is finite.

For $\theta>0$ and $z$ in $(0,1), \Psi(\theta, z)$ is finite, and its coefficient in (24) vanishes for $\theta=u(z)$ when $z$ is in $(0,1)$. So for $0<z<1$,

$$
C(0, z)=(u(z)-\lambda) \varphi^{\prime}(u(z)) / u(z) .
$$

Set $\Gamma(z)=\sum_{n=0}^{\infty} \gamma_{n} z^{n}(|z|<1)$; then using generating functions, and recalling the definition of $\psi(\theta, z)$ after (22) above, equation (3) can be written as

$$
\Gamma(z)+\frac{\mu^{2}}{1-z}=\int_{0}^{\infty} x d_{x} C(x, z)=-\lim _{\theta \downarrow 0} \frac{\partial}{\partial \theta} \Psi(\theta, z) .
$$

The evaluation of this limit (which exists for $z$ in $(0,1)$ because

$$
\varphi^{\prime \prime}(0)=\lim _{\theta \downarrow 0} E\left[W_{0}^{2} e^{-\theta W_{0}}\right]=\sigma^{2}+\mu^{2}
$$

is finite) leads to

$$
\Gamma(z)+\frac{\mu^{2}}{1-z}=\frac{C(0, z)-\varphi^{\prime}(0)+\lambda \varphi^{\prime \prime}(0)}{\lambda(1-z)}+\frac{\varphi^{\prime}(0)\left(1+\lambda z \beta^{\prime}(0)\right)}{\lambda(1-z)^{2}},
$$

that is

$$
\Gamma(z)=\frac{\sigma^{2}}{1-z}-\frac{\mu z(1-\tau)}{\lambda(1-z)^{2}}+\frac{C(0, z)}{\lambda(1-z)} .
$$

Recall that $\sum_{n=0}^{\infty} C_{n} z^{n}=C(0, z)$, that is

$$
C_{n}=C_{n}(0)=\int_{W_{n}=0} W_{0} \boldsymbol{P}\left(d W_{0}, d W_{n}\right),
$$

and set $\sum_{n=0}^{\infty} \rho_{n} z^{n}=\Gamma(z) / \sigma^{2}$. Then (26) yields (18) by equating coefficients of powers of $z$. It thus remains only to demonstrate (19), which we now do by means of the general form of Lagrange's equation for the reversion of series (e.g. [5] p. 125).

First, note that the function $\chi(\theta)=(\theta-\lambda) \varphi^{\prime}(\theta) / \theta$ is an analytic function of $\theta$ in a neighbourhood of $\theta=\lambda$ with $\chi(\lambda)=0$. Next, the function $g(\theta)=(\lambda-\theta) / \lambda \beta(\theta)$ is an analytic function of $\theta$ in a neighbourhood of $\theta=\lambda$, and its derivative there is $g^{\prime}(\lambda)=-1 / \lambda \beta(\lambda) \neq 0$. Therefore the inverse function $v(z)$ such that $v(0)=\lambda$ and $z=g(v(z))$ for $z$ in a neighbourhood $\mathscr{N}_{0}$ of $z=0$ exists and is unique; since $v(z)=u(z)$ for real positive $z$ 
and $z$ in $\mathscr{V}_{0}, v(z)$ is the analytic continuation of $u(z)$ to the whole of $\mathscr{N}_{0}$. Since $C(0, z)=\chi(u(z))$, Lagrange's formula yields for all $z$ in some neighbourhood of $z=0$

$$
\sum_{n=1}^{\infty} C_{n} z^{n}=C(0, z)=\sum_{n=1}^{\infty} \frac{z^{n}}{n !}\left[D^{n-1}\left(\chi^{\prime}(\theta)[-\lambda \beta(\theta)]^{n}\right)\right]_{\theta=\lambda} \quad\left(D \equiv \frac{d}{d \theta}\right) .
$$

Substituting for $\chi(\theta)$, equating coefficients of powers of $z$, and simplifying, yields (19) and completes the proof of the theorem.

We remark that, apart from (19) (and hence Theorem 5 below), the present results are obtained by real variable arguments.

It may also be noted that the same method can be used to deduce comparable results for higher moments. For example, with $0<z<1$, if $\Gamma^{(2)}(z)=\sum_{n=0}^{\infty} \gamma_{n}^{(2)} z^{n}$, where $\gamma_{n}^{(2)}+\left(\sigma^{2}+\mu^{2}\right)^{2}=E\left[W_{0}^{2} W_{n}^{2}\right]$, then setting

$$
C_{n}^{(2)}(x)=\int_{W_{n} \leqq x} W_{0}^{2} P\left(d W_{0}, d W_{n}\right),
$$

equations analogous to (23), (24) and (25) follow, with $\varphi^{\prime}(\theta)$ replaced by $-\varphi^{\prime \prime}(\theta)$. It is of course now required to assume that $E\left[S^{5}\right]$ is finite, while the analogue of Theorem 4 (and presumably of Theorem 2 also, if such an analogous representation can be found) would speak of the finiteness of the sixth moment.

Similarly, the same techniques can be used to develop comparable formulae for the system $E_{k} / G / 1$.

The next theorem gives an exact expression for $\sum_{n=0}^{\infty} \rho_{n}$ in the system $M / G / 1$; the result given in (28) should be compared with the approximation at (14) above, noting that in (28) $E[-U]=(1-\tau) / \lambda$. then

THEOREM 4. $\sum_{n=0}^{\infty} \rho_{n}$ is finite if and only if $\mu_{4}=E\left[S^{4}\right]<\infty$. If $\mu_{4}<\infty$,

$$
\begin{aligned}
1+2 \sum_{n=1}^{\infty} \rho_{n} & =\frac{1+\tau}{1-\tau}+\frac{\lambda\left(-\varphi^{\prime \prime \prime}(0)-\mu \varphi^{\prime \prime}(0)\right)}{(1-\tau) \sigma^{2}} \\
& =\frac{1+\tau}{1-\tau}+\frac{\lambda\left(E\left[W^{3}\right]-E[W] E\left[W^{2}\right]\right)}{(1-\tau)\left(E\left[W^{2}\right]-E[W] E[W]\right)} .
\end{aligned}
$$

Proor. The first part of the theorem is known via Theorem 2, but it can also be established by finding the conditions under which

$$
\sum_{n=0}^{\infty} \rho_{n}=\lim _{z \uparrow 1} \Gamma(z) / \sigma^{2}
$$

is finite. Replacing $C(0, z)$ in (26) by (25) and applying l'Hôpital's rule three times we find (essentially) equation (27), provided that

$$
\lim _{z \uparrow 1} \varphi^{\prime \prime \prime}(u(z))=\lim _{\theta \downarrow 0} \varphi^{\prime \prime \prime}(\theta)
$$

is finite. The algebraic details are omitted. 
We observe that by the Cauchy-Schwartz inequality, the second term in (28) is non-negative, and therefore the first term in (28) is a lower bound for the left hand side of (27).

CoRollary 4.1. If $\left\{w_{1}, \cdots, w_{N}\right\}$ is an observed sequence of waiting times in an $M / G / 1$ queueing system in equilibrium, and if $\mu_{4}<\infty$, then for $N$ large,

$$
N^{-1} \operatorname{var}\left(\sum_{n=1}^{N} w_{n}\right) \approx \frac{(1+\tau) \sigma^{2}+\lambda\left(E\left[W^{3}\right]-E[W] E\left[W^{2}\right]\right)}{(1-\tau)} .
$$

PROOF. $\operatorname{var}\left(\sum_{n=1}^{N} w_{n}\right)=N \sigma^{2}+2 \sum_{n=1}^{N-1}(N-n) \rho_{n} \sigma^{2} \sim N \sigma^{2}\left(1+2 \sum_{1}^{\infty} \rho_{n}\right)$, the approximation holding provided the infinite series converges, which is the case when $\mu_{4}<\infty$.

\section{COROLlaRY 4.2 For $\tau$ near 1 ,}

$$
1+2 \sum_{1}^{\infty} \rho_{n}=\frac{2 \lambda^{2} \mu_{2}}{(1-\tau)^{2}}+\frac{1+\tau}{1-\tau}+\frac{2 \lambda \mu_{3}}{3 \mu_{2}(1-\tau)}+O(1) .
$$

Proof. From the Pollaczek-Khintchine equation (16) we deduce that

$$
E\left[W^{3}\right]=\frac{3 \lambda^{3} \mu_{2}^{3}}{4(1-\tau)^{3}}+\frac{\lambda^{2} \mu_{2} \mu_{3}}{(1-\tau)^{2}}+\frac{\lambda \mu_{4}}{4(1-\tau)} .
$$

Recalling (17) and substituting in (28) leads to (29).

In passing we note that (15) in the case of an $M / G / 1$ system yields

$$
1+2 \sum_{1}^{\infty} \rho_{n} \approx 1+\frac{2\left(\lambda^{2} \mu_{2}-\tau^{2}+1\right)}{(1-\tau)^{2}}=\frac{2 \lambda^{2} \mu_{2}}{(1-\tau)^{2}}+\frac{2(1+\tau)}{I-\tau}+1
$$

in which the leading term agrees with that of (29) for $1-\tau=o(1)$.

These corollaries are important in indicating the relative sizes of samples required for a Monte Carlo simulation study of the mean waiting time in an $M / G / 1$ queue (cf. [6] and [10]). In particular, in order to obtain the same accuracy (without taking into consideration the changing variance) the sample size should vary as $(1-\tau)^{-2}$ (approximately), while in order to estimate the mean to the same absolute accuracy the size should vary as $(1-\tau)^{-4}$. Presumably the orders of magnitude are similar for many server systems, but these questions lie beyond this paper.

\section{Behaviour of $\rho_{n}$ in $M / G / 1$ with $\tau$ near 1}

The main object of this section is to use (18) and (19) (which otherwise do not appear to have any particular advantage to offer over (25) and (26)) to establish 
THEOREM 5. For the family of stable $M / G / 1$ queueing systems with given service time distribution $B(\cdot)$ and arrival rate $\lambda$ such that $0<1-\tau<\delta<1$ and $\mu_{3}=\int_{0}^{\infty} x^{3} d B(x)<\infty$, the serial correlation coefficients $\rho_{n}$ of a stationary sequence of waiting times satisfy

$$
\left|1-A n(1-\tau)^{2}-\rho_{n}\right|<K(1-\tau)^{3}
$$

for any finite set of integers $n=1, \cdots, n_{1}$, where $A=2 / \lambda^{2} \mu_{2}$ and $K$ is a constant depending on $n_{1}$.

Before proving this theorem, we note that in $M / M / 1 A=\tau^{-2}$, so for $\tau$ near $1,(30)$ reduces to (1) as conjectured by Craven [6]. By comparing (30) with known ([12], [13]) heavy traffic results for the stationary $G I / G / 1$ queue, we in turn are led to conjecture that in $G I / G / 1$ the coefficients $\rho_{n}$ satisfy

$$
\rho_{n}=1-n \operatorname{var}\left(U_{t}\right) \kappa^{2} / 2+O\left(\kappa^{3}\right)
$$

for a finite set of integers $n$, where $\kappa=2 E\left[-U_{t}\right] / \operatorname{var}\left(U_{t}\right)$ and $U_{t}=S_{t}-T_{t}$ as defined at the outset; the approximation should hold provided that $\kappa E\left[-U_{t}\right]$ is small.

Proof of Theorem 5. We have to establish that (30) is true for $\tau$ arbitrarily close to one. Comparing (30) with (18) and (17), the coefficient of $-n$ in the exact expression for $\rho_{n}$ in (18) is

$$
\frac{\mu(1-\tau)}{\lambda \sigma^{2}}=\frac{2(1-\tau)^{2}}{\lambda^{2} \mu_{2}+4(1-\tau) \lambda \mu_{3} / 3 \mu_{2}}=\frac{2(1-\tau)^{2}}{\lambda^{2} \mu_{2}}+O\left((1-\tau)^{3}\right),
$$

so (30) will be proved if we can show that for appropriate $n$ and $\lambda$, $\left(\sum_{r=1}^{n} C_{r}\right) / \lambda \sigma^{2}=O\left((1-\tau)^{3}\right)$. Since $\sigma^{2}=O\left((1-\tau)^{2}\right)$, it suffices to show that $\sum_{1}^{n} C_{r}=O(1-\tau)$.

Referring to (19), $C_{r}$ is a function of $\varphi^{\prime}(\theta)$ and $\beta(\theta)$ and of their derivatives at $\theta=\lambda$ : consider in particular the function

$$
\omega(\theta, \lambda)=-\frac{\lambda\left(1-\beta(\theta)+\theta \beta^{\prime}(\theta)\right)}{\theta(\theta-\lambda+\lambda \beta(\theta))^{2}}=\frac{\varphi^{\prime}(\theta)}{\theta(1-\tau)},
$$

the former equality defining a function $\omega(.$, .) for all $\theta$ and $\lambda$ in a range $\lambda_{1} \leqq \theta, \lambda \leqq \lambda_{2}$, the latter equality holding by the Pollaczek-Khintchine equation (16) above only for $\lambda$ such that $0<1-\tau<1$. For $\varepsilon>0$, provided $0<\lambda<(1+\varepsilon) / \mu_{1}=\lambda_{2}$ say, $\omega(\theta, \lambda)$ is analytic in $R l(\theta)>\theta_{0}=\theta_{0}(\varepsilon)$, and $\beta(\theta)$ is analytic in $R l(\theta)>0$. Further, $\varepsilon$ can be chosen so small that $\theta_{0}(\varepsilon)<\lambda_{1}=(1-\delta) / \mu_{1}$. Then the set $L=\left\{(\theta, \lambda): \theta=\lambda, \lambda_{1} \leqq \lambda \leqq \lambda_{2}\right\}$ is a compact subset of the (open) domain on which $\omega(.,$.$) and \beta(\cdot)$ are analytic, and therefore on $L$, a finite set of derivatives of finite order of finite products of $\omega$ and $\beta$ is uniformly bounded. $\left\{C_{r} /(1-\tau), r=1, \cdots, M\right\}$ is such a set, 
defined on a subset of $L$, so $C_{r}=O(1-\tau)$ as required, and this proves the theorem.

In discussing the transient behaviour of a queue in heavy traffic, Kingman $\left([12]\right.$, p. 386) notes that $\kappa^{-2}$ is a measure of the time required for a $G I / G / 1$ system to settle down to its equilibrium state. (30) is a special case of (31) and shows that for $M / G / 1$ a similar conclusion is reached by examining the serial correlation coefficients.

\section{The nature of $\rho_{n}$ in the queue $M / M / 1$}

Application of the results of the foregoing sections to the simple queue $M / M / 1$ with $B(x)=1-e^{-\nu x}, \nu>0$ such that $\tau=\lambda / v<1$, gives moderately tractable formulae. We list the results of the algebra below, and then after numerical illustrations, find a representation for $\rho_{n}$.

$$
\varphi(\theta)=(1-\tau)\left(1+\frac{\lambda}{\theta+\nu-\lambda}\right), \quad \mu=\frac{\tau^{2}}{\lambda(1-\tau)}, \quad \sigma^{2}=\frac{\tau^{3}(2-\tau)}{\lambda^{2}(1-\tau)^{2}} .
$$

The coefficient of $-n$ in $(18)$ is $(1-\tau)^{2} / \tau(2-\tau)$.

$$
\begin{aligned}
u(z) & =\frac{\lambda(1+\tau)}{2 \tau}\left(\sqrt{1-a z}-\frac{1-\tau}{1+\tau}\right), \text { where } a \equiv \frac{4 \tau}{(1+\tau)^{2}}, \\
C(0, z) & =\frac{1-\tau^{2}}{2 \lambda(1-z)^{2}}\left(\sqrt{1-a z}+\frac{2 \tau z}{1+\tau}-1\right),
\end{aligned}
$$

so

$$
\begin{aligned}
& C_{n}=\frac{1-\tau^{2}}{2 \lambda}\left(\frac{2 \tau n}{1+\tau}+\sum_{r=1}^{n}(n+1-r)\left(\begin{array}{l}
\frac{1}{2} \\
r
\end{array}\right)(-a)^{r}\right) ; \\
& 1+2 \sum_{n=1}^{\infty} \rho_{n}=\frac{1+\tau}{1-\tau}+\frac{2 \tau(3-\tau)}{(2-\tau)(1-\tau)^{2}} .
\end{aligned}
$$

The table below contains values of $\rho_{n}$ for $n=0(1) 10$, with $\tau=0.5,0.8$, and $\mathbf{0 . 9}$. For the last value, the approximation from (1) is also given.

In a remark following Theorem 3 we noted that $\left\{\rho_{n}\right\}$ is a convex sequence in $M / G / 1$, and asserted that in $M / M / 1$ it is completely monotonic, a necessary and sufficient condition for which is that the terms $\rho_{n}$ be expressible

$$
\rho_{n}=\int_{[0,1]} t^{n} m(d t)
$$

where $m(\cdot)$ is a bounded non-negative measure on $[0,1]$ (e.g. $[19]$ p. 108). This assertion about $\left\{\boldsymbol{\rho}_{n}\right\}$ will be proved by finding such a measure $m(\cdot)$.

Recall from Lemma 1 that $C_{n} \uparrow \mu W(0)=\mu(1-\tau)$, so it suffices to consider the second differences of $\left\{C_{n}\right\}$ which are expressible (from (32)) as 
TABLE 1

Some serial correlation coefficients of waiting times in $M / M / 1$

\begin{tabular}{lrrrr}
\hline & & & \multicolumn{2}{c}{$\tau=0.9$} \\
\cline { 3 - 5 } & $\tau=0.5$ & $\tau=0.8$ & \multicolumn{1}{c}{ Exact } & Approx. \\
\hline 0 & & & 1.00000 & 1.000 \\
1 & 1.00000 & .00000 & .99043 & .990 \\
2 & .77778 & .96296 & .98125 & .980 \\
3 & .61728 & .92913 & .97237 & .970 \\
4 & .49657 & .89778 & .96376 & .960 \\
5 & .40345 & .86850 & .95539 & .950 \\
6 & .33032 & .84098 & .94722 & .940 \\
7 & .27214 & .81499 & .93924 & .930 \\
8 & .22536 & .79037 & .93144 & .920 \\
9 & .18745 & .76696 & .92381 & .910 \\
10 & .15650 & .74467 & .91630 & .900 \\
$\sum_{n=0}^{10} \rho_{n}$ & .13110 & .72323 & 10.52122 & \\
$\Sigma_{0}^{\infty} \rho_{n}$ & 4.59794 & 9.33957 & 181.81818 & \\
\hline
\end{tabular}

$$
\begin{aligned}
\frac{\lambda^{2} \sigma^{2}}{1-\tau^{2}}(-)^{3} \Delta^{3} \rho_{n} & =-\frac{\lambda}{1-\tau^{2}} \Delta^{2} C_{n}=\frac{1}{2}\left(\begin{array}{c}
\frac{1}{2} \\
n+2
\end{array}\right)(-a)^{n+2} \\
& =\frac{\Gamma\left(n+\frac{3}{2}\right) a^{n+2}}{4 \Gamma(n+3) \Gamma\left(\frac{1}{2}\right)} \quad(\Gamma(\cdot) \text { now denoting the gamma function) } \\
& =\frac{a^{n+2}}{4 \Gamma\left(\frac{1}{2}\right) \Gamma\left(\frac{3}{2}\right)} \cdot \frac{\Gamma\left(n+\frac{3}{2}\right) \Gamma\left(\frac{3}{2}\right)}{\Gamma(n+3)}=\frac{a^{n+2}}{2 \pi} \int_{0}^{1} u^{n+\frac{1}{2}}(1-u)^{\frac{1}{2}} d u \\
& =\frac{1}{2 \pi} \int_{0}^{a} t^{n+\frac{1}{2}}(a-t)^{\frac{1}{2}} d t .
\end{aligned}
$$

Thus, since $0<a<1$, we find

$$
\rho_{n}=\frac{(1-\tau)^{3}(1+\tau)}{2 \pi \tau^{3}(2-\tau)} \int_{0}^{a} t^{n} \frac{t^{\frac{1}{2}}(a-t)^{\frac{1}{2}}}{(1-t)^{3}} d t
$$

which is of the required form (33).

By using (34) to form the spectral density function we can show that it is monotonic decreasing on $(0, \pi)$.

Substituting $t=a u$ in $(\mathbf{3 4})$, we readily deduce that

$$
(1+\tau)^{6} \geqq \frac{8\left(1-\tau^{2}\right)^{3} a^{n} \Gamma\left(n+\frac{3}{2}\right) \Gamma\left(\frac{3}{2}\right)}{\pi \tau(2-\tau) \rho_{n} \Gamma(n+3)} \geqq(1-\tau)^{6},
$$

and since the gamma function has the property that for fixed $b$,

$$
n^{b} \Gamma(n) / \Gamma(n+b) \rightarrow 1
$$$$
(n \rightarrow \infty)
$$ 
(e.g. [5] p. 212), it follows that

$$
\rho_{n}=O\left(a^{n} n^{-\frac{3}{2}}\right) \quad(n \rightarrow \infty) .
$$

Thus the power series $\sum \rho_{n} z^{n}$ has radius of convergence $1 / a$, which is the same as the radius of convergence of $\sum\left(W_{n}(x)-W(x)\right) z^{n}$, where

$$
W_{n}(x)=P\left[W_{n} \leqq x \mid W_{0}=0\right]
$$

(this is proved in [4]). Presumably this observation is a special case of a similar property of the more general system $G I / G / 1$, namely, we suggest that the radii of convergence of

and of

$$
\sum \operatorname{cov}\left(W_{0}, W_{n}\right) z^{n}
$$

are the same.

$$
\sum\left(W_{n}(x)-W(x)\right) z^{n}
$$

\section{Acknowledgements}

It is a pleasure to thank Professor D. G. Kendall for his comments on an earlier version of this paper and related work, which has been done during the tenure of an Australian Canteen Services Trust Fund Overseas Scholarship and completed as a B.O.R. Research Fellow of Selwyn College, Cambridge.

\section{References}

[1] V. E. Benes, 'The covariance function of a simple trunk group, with applications to traffic measurements', Bell System Tech. J. 40 (1961), 117-148.

[2] H. Breny, 'Quelques propriétés des files d'attentes où les clients arrivent en grappes', Mem. Soc. Roy. Sci. Liège 6 (1961), No. 4.

[3] H. Breny, 'Non-indépendence et estimation par la méthode de Monte Carlo: un exemple simple', Bull. Soc. Roy. Sci. Liège 30 (1961), 247-257.

[4] C. K. Cheong and C. R. Heathcote, 'On the rate of convergence of waiting times' J. Aust. Math. Soc. 5 (1965), 365-373.

[5] E. T. Copson, An Introduction to the Theory of Functions of a Complex Variable (Oxford U.P., 1935).

[6] B. D. Craven, 'Serial dependence of a Markov process', J. Aust. Math. Soc. 5 (1965), $299-$ 314.

[7] D. J. Daley, 'Stochastically monotone Markov chains' (in preparation).

[8] W. Feller, An Introduction to Probability Theory and its Applications, Vol. II (Wiley, 1966).

[9] G. S. Fishman and P. J. Kiviat, 'Spectral analysis of time series generated by simulation models', RAND Memorandum RM-4393-PR (1963).

[10] J. R. Jackson, 'Distributions d'échantillonnage du temps moyen d'attente dans une file', Bull. Soc. Roy. Sci. Liège 30 (1961), 243-246.

[11] J. Kiefer and J. Wolfowitz, 'On the characteristics of the general queueing process, with applications to random walks', Ann. Math. Statist. 27 (1956), 147-161.

[12] J. F. C. Kingman, 'On queues in heavy traffic', J. Roy. Statist. Soc. B 24 (1962), $383-392$. 
[13] J. F. C. Kingman, 'The heavy traffic approximation in the theory of queues'. pp. 137 - 169 of Proceedings of the Chapel Hill Symposium on Congestion Theory (University of North Carolina Press, 1965).

[14] P. Le Gall, Les Systèmes avec ou sans Attente et les Processus Stochastiques, Tome I (Dunod, 1962).

[15] P. M. Morse, 'Stochastic properties of waiting lines', Operat. Res. 3 (1955), 255-261.

[16] N. U. Prabhu, Queues and Inventories: A Study of their Basic Stochastic Processes (Wiley, 1965).

[17] J. Riordan, Stochastic Service Systems (Wiley, 1962).

[18] T. L. Saaty, Elements of Queueing Theory with Applications (McGraw-Hill, 1961).

[19] D. V. Widder, The Laplace Transform (Princeton U.P., 1941).

[20] A. Zygmund, Trigonometrical Series (Cambridge U.P., 1959).

[21] D. J. Daley, 'The correlation structure of the output process of some single server queuing systems' (submitted to Ann. Math. Statist., 1967)

\section{Statistical Laboratory}

Cambridge, England 